\title{
TRANSICIONES A LA VIDA ADULTA: GENERACIONES Y CAMBIO SOCIAL EN CHILE
}

\author{
ÓSCAR DÁVILA* \\ FELIPE GHIARDO **
}

\begin{abstract}
RESUMEN
La propuesta de investigación, como estado de avance que se presenta, intenta explorar los procesos de generación de sujetos adultos. Cada sociedad distingue clases de edad que encarnan el período de paso y sobre las cuales se elaboran imaginarios culturales. Si se asume que los modos de constitución de sujetos adultos han cambiado, que los pasos y, sobre todo, las edades a las que se van produciendo los diferentes hitos que marcan el proceso han ido variando, pero que existen diferencias importantes dependiendo de cuestiones estructurales y biográficas.

Este campo de interrogantes es donde se ubica la indagatoria, y su objeto de análisis son las modalidades que adquieren los procesos de generación de sujetos adultos. Y a modo de hipótesis general, se plantea que «existen diferencias cualitativas en los modos en que se articulan los procesos de transición a la vida adulta, dependiendo de la posición social y núcleo generacional, con diferencias generacionales de acuerdo a los niveles socioeconómicos, de género y familiar».
\end{abstract}

PALABRAS CLAVE: JUVENTUDES, CAMBIO SOCIAL, GENERACIONES

* Trabajador social. Centro de Estudios Sociales CIDPA.

E-Mail: oscar@cidpa.cl.

** Licenciado en sociología. E-Mail: felipe@cidpa.cl.

El texto corresponde a un avance de investigación Proyecto FONDECYT 1120926. 


\title{
TRANSIÇÕES PARA A VIDA ADULTA: GERAÇÕES E MUDANÇA SOCIAL NO CHILE
}

\begin{abstract}
RESUMO
A proposta da pesquisa, conforme o estágio de avanço atual, tenta explorar os processos de geração de sujeitos adultos. Cada sociedade define grupos etários que representam a etapa de passagem, sobre os quais os imaginários culturais são elaborados. Supõe-se que os modos de constituição de sujeitos adultos - bem como as etapas, e acima de tudo, as idades em que ocorrem os diferentes ritos que marcam o processo - têm variado, mas existem diferenças significativas por conta de questões estruturais e biográficas.

É neste campo de indagações que se situam os problemas da pesquisa, sendo que as modalidades dos processos de geração de sujeitos adultos constituem seu objeto de análise. Como hipótese geral, sugere-se que «existem diferenças qualitativas nos modos em que se articulam os processos de transição para a vida adulta, dependendo da posição social e do núcleo geracional, com diferenças geracionais, conforme os níveis socioeconômicos, de gênero e familiares».
\end{abstract}

PALAVRAS CHAVE: JUVENTUDES, MUDANÇA SOCIAL, GERAÇÕES

\section{TRANSITIONS INTO ADULTHOOD: GENERATIONS AND SOCIAL CHANGES IN CHILE}

\begin{abstract}
The research proposal presented in this article is part of an ongoing status, and attempts to explore the process of the generation of adult subjects. Each society distinguishes age classes that embody the crossing period on which a cultural imaginary was prepared. Assuming that the modes of incorporation of adult subjects have changed, that the steps and, above all, the ages at which they occur mark different milestones and the processes have varied, there are significant differences depending on structural and biographical issues.

This field is where you will find questions, and analyze the patterns which the generation of adult subjects is acquiring. As a general hypothesis, it suggests that «there are qualitative differences in the ways in which they articulate the transition to adulthood, depending on the social and generational core with generational differences according to socioeconomic levels of gender and family».
\end{abstract}

KEY WORDS: YOUTH, SOCIAL CHANGE, GENERATIONS 


\section{NUEVOS MODOS DE «GENERACIÓN» DE JUVENTUDES}

UNA DE LAS NOCIONES que mejor sintetiza cómo se piensa el actual período histórico es la de cambio. Captar la magnitud y la dirección de esos cambios ha sido un eje fundamental para las ciencias sociales, por lo cual nos interesa explorar una entrada en particular: los procesos de generación de sujetos adultos. Se trata de un componente fundamental para todo grupo humano; de ahí el carácter ritual que adopta en la mayor parte de las culturas primitivas (Metzeltin, 2000). Estudios antropológicos revelan que cada sociedad distingue clases de edad que encarnan el proceso de constitución de sujetos adultos y que sobre ellas se elaboran imaginarios culturales. Feixa distingue cinco modelos históricos, cuyas variaciones han dependido de los grados de complejidad económica y política de las sociedades. A mayor complejidad — dice Feixa-, mayor probabilidad de «moratoria social» (Feixa, 1999). ${ }^{1}$

En las sociedades modernas industriales los procesos de conformación de sujetos autónomos se vuelven más complejos. Entre los estudiosos del tema hay consenso en que esta mayor complejidad responde a las crecientes necesidades de especialización que empezó a requerir el modo de producción capitalista y la organización racional del Estado. De ahí que su estructura quedara definida por el tránsito entre tres instituciones: escuela, trabajo y familia; y de ahí también las diferencias de forma que adopta el proceso dependiendo de la posición que se ocupara en las estructuras de clases y de sexo.

En las primeras décadas del siglo XX se empiezan a suceder los intentos por caracterizar las nuevas etapas en el ciclo de vida que estaban generando estas nuevas formas cada vez más extensivas de generación de sujetos. Uno de los conceptos con que se intentó dar cuenta del nuevo período de tránsito hacia la adultez fue el de adolescencia, descrito como un período de «moratoria social» definido, fundamentalmente, por una serie de cambios biológicos y disposiciones psicológicas (cf. Erikson, 1971). En la época de postguerra, las transformaciones estructurales, sobre todo en educación, revelaron los límites del concepto de adolescencia y moratoria social, muy asociados a la situa-

1 Feixa llama púberes a la condición de moratoria - casi inexistente - en las sociedades primitivas; efebos a los de la sociedad antigua; mozos a la juventud en el antiguo régimen; muchachos a los jóvenes de la sociedad industrial y juventud a los de la sociedad postindustrial (Feixa, 1999). 
ción de los estudiantes de sectores medios y altos. El período definido por el proceso de constitución de sujetos adultos adquirió nuevo nombre: la condición juvenil. Con este concepto se denominó un período vital hasta ese entonces inédito, distinto a la adolescencia, producido básicamente por las transformaciones en las estructuras familiares (Feixa, 1999), cambios institucionales introducidos por el sistema de Estado de Bienestar, la ampliación en los años de escolaridad de las nuevas generaciones y las transformaciones en el sistema productivo.

La irrupción de la juventud como condición social, como actor histórico y como símbolo cultural marcaría el resto del siglo XX. Hobsbawm le dedica un capítulo entero a lo que define como la «juvenilización» de la sociedad occidental (cf. Hobsbawm, 2001). Como señala Beck, el funcionamiento mismo del Estado de Bienestar que se instala en la posguerra activó una creciente tendencia individualizante que sería el motor de lo que define como un proceso de «destradicionalización de las formas de vida de la sociedad industrial». Las concepciones sobre el trabajo y la «carrera estable» perdieron terreno con la cada vez mayor flexibilidad laboral. La incorporación cada vez más fuerte de las mujeres al mundo de la educación y el trabajo, la ampliación de los espacios de participación política de las mujeres, los discursos feministas y los nuevos métodos para regular la fecundidad fueron cambiando el patriarcalismo tradicional en las relaciones de género y el amor (Beck y Beck-Gersheim, 2001), crearon un contexto para la emergencia de nuevas formas de estructuras familiares que ciertamente fueron modificando las modalidades de construcción de autonomías y familia independientes tanto de las mujeres como de los hombres (Beck, 1998).

Todos son elementos que introducen nuevas tensiones y nuevos elementos culturales que se ponen en juego en el tránsito a la adultez. Se trata de nuevos modos de «generación» de juventudes, propios de un tiempo que exige acoplarse a un nuevo modelo de incorporación al mercado laboral, una nueva realidad socioeconómica en la que las incertidumbres y la necesidad de flexibilidad y adaptación sustituyen las certidumbres y las seguridades que les aportaban los modelos de emancipación y tránsito a la vida adulta de las sociedades industrializadas (Montiel, 2009). Los modos de transición que llegaron a ser «típicos» de las distintas clases en el período de expansión del capitalismo industrial fueron dando paso a formatos variables y con direcciones no siempre predecibles. Dejaron de ser un tipo de «trayectoria lineal» y de final conocido, con un eje estable y estandarizado de 
tránsito desde la educación al trabajo, para dar paso a itinerarios de vida o de tránsito a la vida adulta con modulaciones que escapan a las determinaciones, que admiten líneas de acción múltiples, que siguen dependiendo de las situaciones biográficas de los jóvenes, los capitales que herede y los que logre acumular (Bourdieu, 2000, 1998), pero que no necesariamente tienen un final único y conocido, pues dependen de una variedad de situaciones que muchas veces le cambian el rumbo, que diluyen los proyectos. Algunos autores definen estas nuevas modalidades como «trayectorias reversibles, laberínticas o yo-yo» (López, 2002; Machado, 2002a); otros ponen en duda la real reversibilidad de los procesos (Casal, 2006), pero en ambos casos se remite a una sociedad en que estas formas antes numéricamente reducidas o marginales de construcción de la autonomía e independencia se convirtieron en las predominantes. ${ }^{2}$

Ahora bien, como aclara Joaquim Casal, uno de los estudiosos de estos fenómenos, los cambios en los procesos de construcción de una posición profesional y de construcción de emancipación familiar que definen a la juventud, son procesos que experimentan las sociedades avanzadas en su paso desde la sociedad industrial hacia la sociedad digital e informacional (Casal, 2006). La pregunta es hasta qué punto esta interpretación es replicable cuando lo que se quiere es entender los procesos ocurridos en sociedades distintas a las avanzadas o «centrales». ¿Son homologables las situaciones y transferibles las explicaciones a sociedades como las latinoamericanas, que recién hace unas pocas décadas empezaron a dejar atrás las bases agrarias tradicionales dominantes por tres siglos?, ¿se pueden aplicar los mismos esquemas interpretativos a sociedades en que no hubo un Estado de Bienestar del tipo europeo, sociedades que pasaron por dictaduras militares, sociedades de alguna manera insertas en la economía global desde posiciones que sólo parcialmente permiten entenderlas como parte de la sociedad informacional?

2 Para dar cuenta de estas transformaciones, en Europa se han conformado equipos de investigación internacionales que han realizado estudios comparados para tratar de entender los elementos comunes y las diferencias de estos procesos en los distintos países. El Instituto de Juventud español (INJUVE) ha dedicado números completos de su revista a estudios sobre estas transformaciones en la juventud española y europea en general. Ver en particular Revista de Estudios sobre Juventud del Instituto de la Juventud de España (INJUVE) N56, Nº58 y Nº71. 
En el caso de Chile, hay cambios sociales, demográficos y culturales que inciden sobre los procesos de conformación profesional y conformación de independencias familiares que parecieran apuntar en la misma dirección. Así, por ejemplo, cambios en el sistema de trabajo han impuesto formas cada vez más inestables y flexibles de inserción laboral que sin duda repercuten en los procesos de conformación de independencias económicas y familiares. Algunos estudios han dado cuenta de procesos de «individualización» crecientes de las pautas culturales, sobre todo entre los jóvenes (Lechner, 2004). La ampliación del acceso al sistema educativo, que en las últimas décadas ha avanzado aceleradamente hasta llegar a niveles de cobertura casi universal tanto en la enseñanza básica como en la media, y que en buena medida se debe a la cada vez mayor participación de los sectores populares y de las mujeres en los distintos niveles del sistema escolar. ${ }^{3}$ Por ahí se explica que la población joven tienda a retrasar su autonomía y alargar su condición de dependencia en todos los tramos de edad y en ambos sexos, aunque más marcada aún en las mujeres.

Por su parte, indicadores demográficos muestran que las últimas décadas registran tendencias en el mismo sentido. La fecundidad, por ejemplo, muestra una tendencia general que apunta a la postergación del nacimiento del primer hijo o hija; las tendencias en nupcialidad muestran que la condición de soltero experimenta un alza, mientras los casados y convivientes presentan la tendencia inversa, ${ }^{4}$ lo que se expresa también en la edad promedio en que se contrae matrimonio, que también va en aumento. ${ }^{5}$ Tras estas tendencias en los procesos de

3 En las dos últimas décadas esta tendencia es clara. Todos los niveles del sistema escolar muestran una lenta pero progresiva alza en sus tasas de cobertura. En el caso de la educación superior, por ejemplo, según datos de la Encuesta CASEN, entre 1990 y 2003 su cobertura creció 2,3 veces en toda la población - pasó del $16 \%$ al $37,5 \%$ - e incluso se triplicó entre los jóvenes del $40 \%$ de los hogares de menores ingresos: pasó del $4,4 \%$ al $14,5 \%$, en el caso del primer quintil, y del $7,8 \%$ al $21,2 \%$, en el segundo quintil (Mideplan, 2003).

4 Entre el 2003, 2006 y 2009, los jóvenes solteros pasan del $84 \%$ al $88,6 \%$ y al $92,2 \%$; los casados de $12,8 \%$ al $7,5 \%$ y el 2009 al $4,8 \%$, respectivamente (INJUV, 2010). Hay una fuerte disminución en el número de matrimonios: en 1990 fueron 104.740, bajando a los 66.132 el año 2011 (Servicio de Registro Civil e Identificación de Chile, 2012).

5 Si en 1980 la edad promedio de nupcialidad era de 26,6 años para los hombres y de 23,8 años para las mujeres, en 2010, la edad de los matri- 
conformación de familia están contenidas nuevas formas de identidad femenina, más autónomas e individualizadas, orientadas al logro de una independencia económica y una autonomía afectiva (Montilva, 2007 y 2008).

Todo pareciera indicar que, en términos generales, se puede pensar que se replican tendencias que apuntan en la misma dirección que en los países avanzados, pero análisis más detallados sugieren cierta cautela. El mismo análisis de Lechner sobre la individualización reconoce que este cambio cultural presenta diferencias importantes que dependen, fundamentalmente, de condiciones estructurales (Lechner, 2004). Los estudios sobre las nuevas subjetividades femeninas por lo general se remiten a mujeres estudiantes universitarias (Acevedo, 2007) y profesionales de ciertos sectores (Montilva, 2007 y 2008), lo que de alguna manera sugiere que estas nuevas pautas en cierta medida concentran a sus portadoras en mujeres de estos sectores y condiciones. Desde nuestra propia práctica investigativa hemos podido describir las formas que asumen estos procesos en sucesivas cohortes de población joven (Ghiardo y Dávila, 2008). Mediante el análisis de las encuestas nacionales de juventud, se logró identificar diferencias en los procesos de inserción a la vida productiva y la conformación de pareja y familia. En líneas gruesas, mientras en los sectores de mayores recursos predomina una tendencia hacia la continuidad generalizada desde la escuela directamente a la educación terciaria, sin diferencias entre sexos, en los sectores medios se da una mayor dispersión en los rumbos de las trayectorias, con un grupo que sigue estudios superiores y otro relativamente similar que entra a trabajar, y una diferencia importante entre hombres y mujeres tanto en lo que respecta al trabajo como a los quehaceres del hogar. Lo anterior se expresa también en diferencias correlativas en los procesos de autonomía residencial y familiar. Mientras en los sectores de mayores ingresos, tanto hombres como mujeres presentan una tendencia a retrasar la independencia económica y residencial y a posponer la maternidad/paternidad, en los sectores medios y bajos, pero sobre todo en estos últimos, la formación de familia a edades más tempranas es un fenómeno bastante más frecuente. ¿Qué explica estas diferencias?, ¿el hecho de que estos procesos no se produzcan de la misma forma o con la misma

monios sube a los 31,3 años para los hombres y 29,9 años para las mujeres, un alza que es superior en las mujeres que en los hombres $(6,1$ vs 4,7 años, respectivamente). 
fuerza puede hacer pensar en la presencia de bases culturales diferentes que complementen la referencia al solo factor material o socioeconómico?

Lo anterior plantea dos puntos que son relevantes y que fundamentan esta argumentación. En primer lugar, la importancia de los componentes subjetivos que subyacen a las diferentes modalidades de conformación de la autonomía y la independencia. Al análisis de las tendencias a partir de estas bases de datos se le escurre la complejidad de los procesos. Si bien permite describir tendencias generales y compararlas por estratos y grupos, no alcanza a captar los cambios de situación en los trayectos individuales y que son los que le imprimen una dinámica interna de base sociocultural a los procesos de transición. Nuestro propio estudio tiene sus limitaciones, pues si bien acerca a estos componentes desde una perspectiva que investiga las subjetividades implicadas en las trayectorias laborales y su nexo con los procesos de conformación de autonomía e independencia económica, residencial y familiar, su reducción a jóvenes de sectores medios y medio-bajos impide establecer comparaciones entre modalidades o «lógicas» que pudieran obedecer a diferencias socioeconómicas y su relación con las identidades de género, por ejemplo (Ghiardo y Dávila, 2008).

En segundo lugar, el estudio con generaciones jóvenes contemporáneas describe tendencias que informan sobre diferencias entre cursos de vida que coexisten en un mismo período de tiempo, que son contemporáneas, es decir, que muestran su cara sincrónica. La dimensión diacrónica, sin embargo, la que compara entre momentos históricos distintos, permanece como interrogante. Esta ausencia se vuelve fundamental, pues es justamente la comparación diacrónica la que puede alumbrar sobre el carácter de los cambios ocurridos en el campo que se propone investigar. Si un punto común en el discurso social e incluso en el científico es reconocer que en el último tiempo se han producido cambios importantes en los procesos de construcción de autonomías e independencia económica y residencial, si se asume que los pasos y, sobre todo, las edades a las que se van produciendo los diferentes hitos que marcan el proceso también han venido variando, queda la pregunta de hasta qué punto esos cambios son equivalentes para los distintos sectores, grupos y clases sociales. Interpretar este componente de cambio requiere puntos de referencia. De ahí la necesidad de buscar estrategias para hacer comparaciones históricas o, más específicamente, intergeneracionales, pues todo cambio es siempre respecto a formas anteriores. 
Estos puntos son la base para el proceso de investigación. Su objeto de análisis son las modalidades que adquieren los procesos de generación de sujetos adultos. La pregunta es por el tipo de cambios en los procesos de construcción de autonomías e independencias que se han producido en los diferentes sectores de la sociedad. ¿Es posible encontrar elementos culturales, transmitidos intergeneracionalmente en los distintos segmentos sociales, que se pongan en juego en los modos en que se proyecta la construcción de autonomías económicas, residenciales y familiares?, ¿qué papel juegan las diferencias y las relaciones de género en la forma que asumen estos procesos las generaciones jóvenes de los distintos estratos socioeconómicos y que asoman, por ejemplo, en las variaciones que se han observado en la conformación de familia y la fecundidad?

Este campo de interrogantes es donde se ubica el presente proyecto. Su objetivo apunta a explorar los modos en que se articulan los elementos objetivos y subjetivos que subyacen a los procesos de tránsito a la autonomía y la independencia en los sectores de la sociedad. Para eso, la única forma es interrogar las modalidades que asume en los diferentes grupos y clases. La respuesta a estas preguntas supone un ejercicio de análisis en varios niveles. En el nivel estructural, requiere contextualizar los factores sociales y demográficos que han caracterizado los procesos de emancipación y construcción de autonomía desplegados por las generaciones en los estratos o clases sociales. En el nivel subjetivo, requiere rastrear los marcos de discurso ideológico que se despliegan en los modos en que las generaciones de jóvenes ponen en juego al momento de transitar por estos procesos (roles de género, discursos sobre el trabajo, entre otros). A nuestro entender, la comprensión en profundidad de los elementos que están al fondo de los cambios en estos procesos representa una entrada fundamental, pero poco explorada, sobre los procesos sociales y culturales de las últimas décadas.

Adentrarse en un estudio de este tipo significa dar un paso pionero en la comprensión de estos procesos, algo que incluso en Europa permanece como una interrogante. ${ }^{6}$ A partir de estudios sobre la cons-

6 Respecto a este punto, el equipo del GRET de la Universidad Autónoma de Barcelona reconoce que «No disponemos de datos suficientes para medir las modalidades de transición de jóvenes de hace treinta años; por esto los cambios de modalidades que estudiamos siempre tienen un carácter hipotético fundamentado en percepciones contrastadas pero sin 
trucción social de las edades, ${ }^{7}$ y de generación de juventudes se pueden tender interesantes y productivos lazos para la reflexión sobre los procesos históricos de cambio social y cultural, que pueden orientar la implementación de políticas que sirvan para generar soportes a los procesos de construcción de autonomías en las generaciones jóvenes y, a la vez, faciliten la apertura de una conversación que allane el entendimiento intergeneracional.

\section{JUVENTUD, TRANSICIONES, TRAYECTORIAS: GUÍAS PARA ORIENTAR LA BÚSQUEDA}

Para dar cuenta de los procesos de transformación que se investiga, conviene precisar los elementos que orientarán la búsqueda. El trabajo del Grupo de Estudios sobre el Trabajo (GRET), de la Universidad Autónoma de Barcelona, ha venido desarrollando el aporte teórico y metodológico quizá más sistemático para estudiar los procesos contemporáneos de constitución de autonomías. Su formulación parte de un concepto que define a la juventud, más que como una categoría social, como un tramo en las biografías que va desde los inicios de la pubertad hasta la consolidación de la emancipación familiar plena, que asocia especialmente a la inserción profesional y a la emancipación familiar, o más específicamente, el acceso a una vivienda de uso propio, como los dos hitos que introducen la marca quizá más rotunda entre un tramo de vida amarrado a dependencias y otro que se abre a la autonomía. Ambos procesos conforman las dos variables que más determinan lo que define como «modalidades de transición» (Casal et al., 2005 y 2006).

De ese modo, Casal elabora un concepto de juventud que toma elementos de las teorías que entienden la juventud como la adquisición de roles, de teorías de las generaciones, pero para focalizarlas en el proceso de adquisición, enclasamiento y de emancipación familiar. Esta forma de entender los fenómenos de juventud coincide bastante con los conceptos de estructuras de transición y trayectorias sociales

validación numérica [...] El análisis biográfico permite conocer e interpretar las modalidades de transición de los jóvenes y las promociones de adultos o mayores. El estudio en vivo de estas biografías de los mayores sería una aportación de gran interés» (Casal et al., 2006:35).

7 Para una reflexión en torno a la importancia de reflexiones de este tipo, ver el trabajo de Carles Feixa sobre los lineamientos para una antropología de las edades (Feixa, 1991). 
que hemos venido desarrollando de forma paralela en diferentes estudios (Dávila, Ghiardo y Medrano, 2004; Ghiardo y Dávila, 2008).

Las estructuras de transición remiten al tipo de «curso de vida» que resulta al hilar los diferentes hitos que van marcando el paso a la incorporación al trabajo, la formación de pareja y familia, y la adquisición de autonomía residencial. La idea es que si se dibuja su secuencia, su orden y sus tiempos, es posible configurar distintas formas de «hacerse adulto» que tienen dos particularidades: carácter histórico y varían dependiendo de las estructuras sociales y culturales (clase, sexo, etnia). Así, y en términos muy generales, la edad y las formas de hacerse adulto presentan variaciones que dependen de lo que cultural y socialmente se define para cada clase de edad y para cada sexo en cada clase de edad. Las diferencias tienen que ver tanto con el tipo de etapas por las que se pasa, como con los tiempos cronológicos en que ocurren estos cambios de condición (estudios/no estudios; trabajo/no trabajo; no maternidad/maternidad; dependencia/independencia). De ahí el carácter de «estructuras». Las trayectorias sociales, por su parte, analizan otro plano del proceso, el de las posiciones que van ocupando los sujetos en la estructura social. Aquí no es la secuencia que producen las distintas «fases» de generación de autonomías lo que importa, sino las posiciones estructurales y las disposiciones subjetivas que envuelven y a su vez producen esos cambios de condición. Si para el análisis de las transiciones el paso de estudiante a trabajador importa en sí mismo, si la edad en que se produce es un factor que influye en la descripción de la estructura de las transiciones, para las trayectorias importan el grupo social de origen, el nivel de educación alcanzado, el tipo de establecimiento escolar, el título y el tipo de trabajo al que se accede con ese título, la valoración social y simbólica del título obtenido. ${ }^{8}$

Tanto el de estructuras de transición como el de trayectorias sociales son herramientas analíticas para dar cuenta de un proceso unitario. Por lo tanto, sólo adquieren capacidad explicativa cuando se los relaciona. Los cambios en la estructura de las transiciones no se pueden comprender si no es en relación a las trayectorias sociales -así, por ejemplo, la relación entre aumento de años de escolaridad y postergación de la independencia-; ni las trayectorias sociales se pueden

8 Para pensar en términos de trayectorias sociales conviene remitirse a la noción de espacio social, de capitales y, en general, a todo el análisis de clases que desarrolla Pierre Bourdieu (cf. Bourdieu, 2000). 
entender sin considerar los sucesos —maternidad, por ejemplo-y las bases sociales y culturales — significados sobre la maternidad - vinculados a las transiciones - para seguir con el ejemplo, reclusión al hogar o inserción al mundo laboral por razones de maternidad-.

Más allá de las diferencias nominales entre el aparato conceptual que desarrolla el GRET y de las estructuras de transición y las trayectorias sociales, ambos coinciden en un mismo punto: que la exigencia de articular elementos sociales, culturales e históricos remite, en último término, a la necesidad de poner en el centro la biografía. ${ }^{9}$ Toda modulación entre estructuras de transición y trayectorias sociales supone una biografía, una historia de vida protagonizada por un actor individual que tiene constricciones estructurales, pero que es capaz de actuar, moverse (Ghiardo y Dávila, 2008). El mismo análisis propone Casal y su equipo. «El enfoque de las biografías procura - dicenuna triangulación a tres bandas: la sociedad como estructura, los hombres y mujeres como actores y las generaciones como resultantes de procesos históricos de cambio. En definitiva: estructura, acción y historia» (Casal, et al., 2006:11). Su punto de partida es el actor social como sujeto histórico y protagonista principal de la propia vida que articula lo que define como un itinerario vital construido por elecciones y decisiones del individuo, pero bajo determinaciones familiares o del entorno próximo, determinaciones estructurales del contexto amplio, y determinaciones de orden cultural y simbólico. La transición, por lo tanto, queda como una articulación compleja de procesos de formación, inserción profesional y emancipación familiar, que se construye socialmente en un marco sociopolítico específico que determina un sistema político de transición y que, a su vez, está influido por factores sociohistóricos y geopolíticos, pero que no niega la capa-

9 Como propone Casal, «Puede ser que en pleno industrialismo el planteamiento funcionalista fuera atractivo y de interés para la Sociología; para la sociedad convulsa del crecimiento acelerado de los 60 y crisis ideológica de los 70 y económica de los 80 parece que el planteamiento generacionalista también podría resultar atractivo e interesante, sobre todo por el acierto de contraponer lo 'viejo' y lo 'nuevo'. Finalmente, para la sociedad que se lanza hacia la turbulencia y cambio del informacionalismo, parece que el planteamiento biografista también puede resultar atractivo y de interés sobre todo atendiendo al hecho del constructivismo social y las rupturas biográficas en lo público y en lo privado; es decir, tres modos sociales y tres perspectivas de análisis» (Casal et al., 2005:4). 
cidad de acción de los sujetos. De ahí que remita a un tramo biográfico de duraciones y estructura variable, con distintas velocidades y procesos (Casal et al., 2006).

El análisis biográfico se estructura por el cruce entre lo que llama «promociones», que equivale a los patrones demográficos que caracterizan a las distintas generaciones, las edades en que se producen los procesos de transición - equivalente a las estructuras de transicióny las características del período histórico. Lo interesante es que además, al incorporar la dimensión geopolítica, releva un elemento que parece cada vez más relevante: el territorio.

Con este conjunto de herramientas conceptuales se puede abordar un estudio lo suficientemente complejo como para comprender diferentes componentes subjetivos implicados, captar los diversos modos de modulación de las transiciones en las generaciones jóvenes contemporáneas y, a partir de su comparación generacional, tratar de interpretar el sentido de los cambios sociales.

Desde ese punto de vista, y en el actual estado de avance del proceso investigativo, es posible explicitar ciertas hipótesis sobre las cuales se orienta la búsqueda. La principal se refiere a que las diferencias en los modos en que se articulan los procesos de transición a la vida adulta entre los jóvenes están asociadas tanto a las condiciones estructurales (capital escolar, capital económico) como a las pautas culturales de las distintas clases y grupos sociales.

Y de modo más específico, i) En los sectores de menores recursos y más reciente incorporación al sistema escolar secundario y superior, la diferencia generacional en las modalidades de transición a la vida adulta es mayor que en los jóvenes que provienen de familias con padres profesionales. ii) El efecto de la flexibilidad laboral sobre la desestabilización de las modalidades de transición a la vida adulta es mayor en los sectores altos y medios profesionales, con mayor vínculo a trabajos estables, a diferencia de los sectores de bajos recursos, en que la inestabilidad laboral es más bien una constante. iii) Las diferencias generacionales en las modalidades de tránsito hacia la vida adulta dependen de cambios en los imaginarios de género, diferencias que son mayores en las clases medias que en los sectores de bajos recursos. iv) Las diferencias en las pautas de fecundidad que articulan las modalidades de transición a la autonomía e independencia tienen bases culturales que se transmiten intergeneracionalmente y varían dependiendo del sector de pertenencia. 
El objetivo último del proceso investigativo se orienta a construir una tipología para comprender las diversas transiciones juveniles al mundo adulto, identificando dimensiones específicas a nivel de los tránsitos laborales, educacionales, familiares y vitales.

VALPARAÍSO (CHILE), SEPTIEMBRE 2012

RECIBIDO: SEPTIEMBRE 2012

ACEPTADO: NOVIEMBRE 2012

\section{REFERENCIAS BIBLIOGRÁFICAS}

BECK, UlRICH (1998): La sociedad del riesgo. Hacia una nueva modernidad. Barcelona: Paidós.

BECK, U. Y E. BECK-GERSHEIM (2001): El normal caos del amor. Las nuevas formas de la relación amorosa. Barcelona: Paidós.

BOURDIEU, PIERRE (2000): Cuestiones de sociología. Madrid: Istmo.

- (1999): La miseria del mundo. Buenos Aires: FCE.

- (1998): Capital cultural, escuela y espacio social. México: Siglo XXI. (1997): Razones prácticas. Sobre la teoría de la acción. Barcelona: Anagrama.

(1988): La distinción. Criterios y bases sociales del gusto. Madrid: Taurus.

— y JeAn-Claude PASSERON (2003): Los herederos. Los estudiantes y la cultura. Buenos Aires: Siglo XXI.

$-\mathrm{y}-$ (1996): La reproducción. Elementos para una teoría del sistema de enseñanza. México: Fontamara.

CASAL, JOAQUim (2004): «Diez proposiciones sobre juventud». En ROSSANA REGUillo et al.: Tiempo de híbridos. Entresiglos jóvenes MéxicoCataluña. México: IMJ.

(2002): «TVA y políticas públicas sobre juventud». Revista de Estudios de Juventud $\mathrm{N}^{\circ} 59$. Madrid: INJUVE.

—_ (1999): «Juventud, transición y políticas sociales». En: La nueva condición juvenil y las políticas de juventud. Barcelona: Diputación de Barcelona.

—- JOSEP MASJOAN y JORDI PlANAS (1988): «Elementos para un análisis sociológico de la transición a la vida adulta». Política y Sociedad $\mathrm{N}^{\circ} 1$. Madrid: UCM.

—; Maribel García, Rafael Merino y Miguel Quesada (2006): «Cambios en las modalidades de transición de los jóvenes en los países del capitalismo informacional». GRET, Grup de Recerca Educació i Treball. Barcelona: Universidad Autónoma de Barcelona. 
$-\quad-\quad \longrightarrow$ y $-(2005)$ : «Aportaciones teóricas y metodológicas a la sociología de la juventud desde la perspectiva de la transición». GRET, Grup de Recerca Educació i Treball. Barcelona: Universidad Autónoma de Barcelona.

DÁVILA, OSCAR y FELIPE GHIARDO (2008): Trayectorias sociales juveniles. Ambivalencias y discursos sobre el trabajo. Valparaíso: Ediciones CIDPA E INJUV.

— - - y CARLOS MEDRANO (2005): Los desheredados. Trayectorias de vida y nuevas condiciones juveniles. Valparaíso: Ediciones CIDPA.

ERIKSON, ERIK H. (1993): Sociedad y adolescencia. México: Siglo XXI (14 ${ }^{\mathrm{a}}$ edición).

- (1971): Identidad, juventud y crisis. Buenos Aires: Paidós.

FEIXA, CARLES (1999): De jóvenes, bandas y tribus. Barcelona: Ariel.

GHIARDO, FELIPE (2004): «Generaciones y juventud: una relectura desde Mannheim y Ortega y Gasset». Última Década N²0. Valparaíso: Ediciones CIDPA.

HOBSBAWM, ERIC (2001): Historia del siglo XX. Barcelona: Crítica.

LECHNER, NORBERT (2004): «Cultura juvenil y desarrollo humano». Jóvenes, Revista de Estudios sobre Juventud $\mathrm{N}^{\circ} 20$. México: IMJ.

LÓPEZ BLASCO, ANDREU (2002): «De los itinerarios lineales a las trayectorias yo-yo». Ponencia presentada en la Conferencia Europea para Investigadores y Técnicos «Jóvenes y políticas de transición en Europa». INJUVE, Madrid, 6 al 8 de junio.

MACHADO PAIS, JOSÉ (2000): «Las transiciones y culturas de la juventud: formas y escenificaciones». Revista Internacional de Ciencias Sociales No164. París: UNESCO.

MANNHEIM, KARL (1982): «O problema sociológico das gerações». En M. FORACHI: Mannheim. São Paulo: Ática.

- (1944): Diagnóstico de nuestro tiempo. México: FCE.

MARTín CRIAdo, EnRIQue (1998): Producir la juventud. Crítica de la sociología de la juventud. Madrid: Istmo.

Metzeltin, Miguel (2000): «De la lingüística a la antropología omnimedial». Revista Española de Lingüística, Vol. 30, Nº1. Madrid: SEL.

MONTIEL, GonZALO (2009): «Transiciones virtuales en la juventud: una aproximación a la emancipación juvenil en la sociedad de la información». RECERCA, Revista de Pensament $i$ Anàlisi N$^{\circ}$. Castellón de la Plana: Universitat Jaume I de Castellón.

MONTILVA, MAIRA (2008): «Postergación de la maternidad de mujeres profesionales jóvenes en dos metrópolis latinoamericanas». Utopía y Praxis Latinoamericana $\mathrm{N}^{\circ} 41$. Maracaibo: Universidad del Zulia. (2007): «Individualización femenina y cohabitación sin papeles: el caso de las profesionales de Santiago de Chile». KAIROS, Revista de Temas Sociales $\mathrm{N}^{\circ} 20$. San Luis: Universidad Nacional de San Luis. 